OPEN ACCESS

Edited by:

Yi Lu,

Fudan University, China

Reviewed by:

Haksu Kyung,

National Medical Center, South Korea Jinhai Huang,

Affiliated Eye Hospital of Wenzhou Medical University, China Adel Ebraheem, California State University, United States

*Correspondence:

Mengmeng Wang wangmengmg@163.com

Specialty section:

This article was submitted to Ophthalmology,

a section of the journal

Frontiers in Medicine

Received: 19 January 2021 Accepted: 08 June 2021

Published: 02 July 2021

Citation:

Wang M, Corpuz CCC and Zhang F (2021) Shaping Eyeballs by Scleral Collagen Cross-Linking: A Hypothesis for Myopia Treatment. Front. Med. 8:655822.

doi: $10.3389 /$ fmed.2021.655822

\section{Shaping Eyeballs by Scleral Collagen Cross-Linking: A Hypothesis for Myopia Treatment}

\author{
Mengmeng Wang ${ }^{1 *}$, Christine Carole C. Corpuz ${ }^{2}$ and Fengju Zhang ${ }^{3}$ \\ ${ }^{1}$ Hebei Ophthalmology Key Lab, Hebei Eye Hospital, Xingtai, China, ${ }^{2}$ Ifugao State University Eye Center, Alfonso Lista, \\ Philippines, ${ }^{3}$ Beijing Tongren Eye Center, Beijing Tongren Hospital, Capital Medical University, Beijing, China
}

The global prevalence of myopia has brought to the attention of the different eye and vision specialists, who make way to control its progression. Evidence have shown that a proactive reshaping of the eyeball is the core point of myopia developing process, which particularly includes the weakening, thinning, and expanding of the sclera. Thus, the sclera is considered to be a prime target for therapeutic manipulation in halting progressive myopia. In the past decades, corneal collagen cross-linking has been applied in clinical practice for treating aberrant corneal remodeling diseases. In this article, we hypothesize that scleral collagen cross-linking $(\mathrm{SXL})$ has a huge potential in stabilizing myopic process by shaping the eyeball and preventing the aberrant scleral remodeling. In contrast with the current methods of optometry correction, such as physiotherapy, pharmacotherapy, spectacles, contact lenses, refractive surgeries, etc., eyeball-shaping method using $S X L$ is a fundamental intervention which aims at the pathogenesis of progressive visual loss of myopia. Compared with the current posterior scleral reinforcement, the most advantage of SXL is that there is no allotransplant into the myopic eye, which means less expenditure, lower risk, and easier to handle in operating.

Keywords: myopia, sclera remodeling, scleral collagen cross-linking, sub-tenon's injection, staphyloma

\section{INTRODUCTION}

Myopia is the most common cause of visual impairment worldwide (1). It was speculated that the global prevalence of myopia by 2050 will be about 4,758 million (49.8\%), including 277 million (4.0\% of the global population) of high myopia (2). The burden of myopia is tremendous, as adults with high myopia are more likely to develop pathologic myopia (PM) changes, such as posterior scleral staphyloma, choroidal neovascularization, retinal choroidal atrophy, streak, peripheral retinal degeneration, retinal detachment, and finally lead to blindness (3-5). Generally, myopia was believed to be a mismatch of the eye's anatomical axial length and its focal length (6) which was caused by a "multifactorial" mechanism $(7,8)$. Aberrant scleral remodeling is the core point during the myopic process, and has been the goal of any long-term therapy for the persistent vision loss associated with myopia (9). Collagen cross-linking, introduced by Wollensak et al. (10), is an effective approach to increase the biomechanical strength of the corneal and scleral tissue. Corneal collagen cross-linking (CXL) has been applied in clinical practice for treating aberrant corneal remodeling diseases in the past decades (11). Similarly, scleral collagen cross-linking (SXL) has a huge potential in stabilizing myopic process by shaping the eyeball and preventing the aberrant scleral remodeling, such as ocular axial elongation and staphyloma. 


\section{HYPOTHESIS}

We hypothesize SXL as an eyeball-shaping method to prevent the aberrant scleral remodeling during myopic process. Such a theory has not been conceptualized yet, and has not been verified by clinical trials. We reason that the strengthening the integral sclera by SXL could slow down the process of diffuse expansion during eyeball growth; strengthening the equatorial sclera by SXL could limit the axial elongation of the progressive myopic eyeball; partially strengthening the sclera by SXL could block the local bulges which causes staphyloma. Due to its cross-linking effect only on the outer layer of the sclera, SXL is a safe method for the function of the inner tissues underneath the sclera. SXL could be artificially carried out by different cross-linking categories, doses, frequencies, sites according to the current status and prognosis of myopic eyes. For these reasons, it will prevent or halt the progression of myopia in any stage of the disease.

\section{ABERRANT SCLERAL REMODELING OF MYOPIA}

There are strong evidences from clinical and experimental studies indicating that the biochemical and biomechanical properties of the sclera play a major role in the progression of myopia (12). Thinning of the sclera and weakened biomechanical properties, particularly at the posterior pole of the eye, have long been known to be an important feature in the development of high myopia in human and mammalian models (9). It is found that myopic sclera undergoes a proactive reshaping process which includes diameter decrease and fiber gap enlargement of the collagenous fiber bundle in all parts of the myopic eye, particularly in the posterior sclera; this causes retinal fundus complications which adversely affect visual acuity $(9,12)$. Thus, the sclera is considered to be a prime target for therapeutic manipulation in halting progressive myopia (13). Currently, scleral reinforcement is the only available clinical option to slow down scleral bulges (such as in staphyloma), which remain controversial due to its complication risk (14). Thus, given the lack of treatment options, novel clinical solutions are becoming increasingly necessary available to halt progressive scleral remodeling and morbidity of progressive myopia.

\section{PRINCIPLE OF SXL}

Cross-linking is a natural phenomenon in the body following an enzymatic or a non-enzymatic pattern (15). Generally, the phenomenon increases in age (16) or in diabetics (17), who rarely show progressive myopia (18). On the contrary, diminished cross-linking is an important factor in the weakening process of myopic sclera (16) with a significant decrease in Young's modulus (19). It is therefore hypothesized that, instead of natural cross-linking, artificially-induced cross-linking might be used therapeutically to retard axial elongation, thereby reducing the risk of blindness.

Photochemically-induced crosslinkings, which currently include riboflavin/UV light crosslinking and riboflavin/blue light crosslinking, can create additional chemical bonds between collagen fibers by photopolymerization and increase scleral stiffness (20). This photochemically-induced cross-linking is mediated by photooxidation between riboflavin (vitamin B2) and laser light (UV light, $360-370 \mathrm{~nm}$; blue light, $400-500 \mathrm{~nm}$ ). The laser light activates riboflavin into triplet, which in turn produces reactive oxygen species (ROS) and singlet oxygen. ROS reacts with collagen fibril molecules in the corneal stroma and enhances the mechanical strength of cornea by forming new chemical bonds between amino groups of collagen fibril molecules (21). Theoretically, blue light has better penetration ability and lower damage in sclera compared to UV light considering the negative relation between wavelength and penetrating depth in tissue (22).

Chemically-induced crosslinking is another kind of artificially-induced cross-linking, which is usually applied by injecting of chemical crosslinking agents into the sub-Tenon's (sT) space and scleral surface. This kind of crosslinking could represent a simpler way to treat the posterior sclera, thus, avoiding the need for UV light exposure (23). Although there are many compounds that are suitable for industrial and commercial cross-linking purposes, only a limited number of compounds have emerged with potential for scleral stiffening in vivo: glyceraldehyde (24), genipin (25-27), methylglyoxal $(28,29)$ (also called pyruvaldehyde), formaldehyde releasers (FARs) (30), sodium hydroxymethylglycinate (SMG) (31), methylglyoxal (32). Applying the concept of the Maillard reaction (15), these crosslinking agents can be added to the ends of protein molecules and can be further transformed to advanced glycation end products, which are more stable. The resultant covalent collagen cross-links promote enhanced tissue stiffness and resistance to enzymatic degradation (33).

\section{TECHNIQUE OF SXL}

Until now, no protocol of photochemically-induced crosslinking was set up for sclera treatment, while several protocols of corneal collagen cross-linking has been suggested to be used on corneal stroma for the treatment of keratoconus, such as the Dresden protocol $(34,35)$, Siena University protocol (36), Athens protocol (37). Based on the experimental parameters reported in previous studies, the operating procedure of photochemically-induced SXL was generally as follows: after adequate exposure of the target scleral surface, a UV/blue light-source was set up to irradiate directly on the target scleral surface. Specific intensity and energy were performed to ensure that the exposure of UV/blue light on the cornea is below harmful levels. A syringe with a blunt needle allowed an almost continuous supply of the riboflavin photosensitizer solution during the irradiation (38).

Compared with the photochemical procedure, which requires a surgical procedure to access the posterior sclera with a UV light source, injecting into the sub-Tenon's space with chemical crosslinking agents is much simpler $(27,39)$. Like photochemicallyinduced crosslinking, no standardized protocol of chemicallyinduced crosslinking has been set up at present, especially in the parameters of drug concentration and dose (32). However, in 
contrast to photochemically-induced crosslinking, repeated subTenon's injections of crosslinking agents can be performed in different quadrants for several times in the following sequential days, months, or years. Another advantage of chemicallyinduced crosslinking is that, with the help of ultrasound, the chemical crosslinking agents can easily be injected via subTenon's approach, to the posterior globe and even the whole sclera, and could create much larger crosslinked areas than photochemically-induced crosslinking $(33,40)$.

\section{KNOWN FACTS ABOUT SXL}

Photochemically-induced crosslinking has been proven to increase the mechanical strength of scleral collagen in guinea pigs (40), rabbits (41), pigs (10), primates (42), and human sclerae (43). A significant increase in the density and area of collagen fibrils and a significant decrease in the density and area of the interfibrillar spacing can be found in both the equatorial and posterior sclera with either riboflavin/UVA or riboflavin/blue light SXL for 12 months postoperatively (42-44). However, the UV/blue light irradiation with high intensities induces degenerative alterations of scleral cells mainly, in the episcleral and outer scleral layers, which is associated with extracellular matrix degradation, tissue inflammation, hemorrhage, and macrophage infiltration; and in the inner scleral layers, where treatment with high-intensity irradiation induces scleral cell activation with pronounced metabolic activity, and finally results in scleral scarring (45). Not only would the high light intensities be harmful, but also, complicated operations would bring dangerous side-effects on the ocular tissues of the crosslinked eye (46). For example, during the irradiation process, the eyes had to be stretched to expose the target scleral region. Irradiation was largely focused on the equatorial area, thereby inducing possible mechanical injury to ocular tissues. This approach made it impossible to fully expose the posterior sclera that is prone to develop staphyloma (47). Moreover, many other adverse factors were encountered in the past, such as complexity of operating riboflavin/UVA sclera collagen cross-linking, large lesions, difficulty in irradiating the posterior sclera, and difficulty in administering repetitive irradiation (48). Although a minimally invasive therapy has reported to be performed by inserting miniaturelightemitting diodes (LEDs) under the tenon's capsule to the posterior sclera without stretching the eyeball tissues (38), its drawbacks have been put forward, including limited irradiance, risk of thermal tissue damage, and poor flexibility (49).

One potential treatment modality to overcome the above adverse factors of SXL is chemically-induced crosslinking by subTenon's injection of chemical crosslinking agents. This method could serve as a simple and minimally invasive approach to reach the posterior sclera without the need for any specialized UV device or UV irradiation (50). Our research team (39) has successfully used sub-Tenon's injection of $0.5 \%(22.1 \mathrm{mM})$ genipin to decelerate form-deprivation myopia in guinea pigs. Another previous study (33) also found that sub-Tenon's injection of $0.15 \mathrm{ml} 0.5 \mathrm{M}$ glyceraldehyde seven times during 14 days reduced the AXL elongation of rabbit eyes, with no retinal and choroidal affects under light microscopy. It was also found that glyceraldehyde could increase scleral stiffness for at least 8 months in rabbits (24). Compared with glyceraldehyde (seven fold) or methylglyoxal (30 fold), a much lower concentration of genipin is required to achieve a similar scleral stiffening effect after the sub-Tenon's injection, which means that SXL using genipin could be an alternative low-cytotoxic collagen crosslinking agents (32). Moreover, sub-Tenon's injection of FARs (30) and SMG (31) have also shown their effects in halting the progression of scleral elongation seen in myopic eyes, and would be potential cross-linking compounds. Nevertheless, it should be mentioned that the safety of chemically-induced crosslinking was not fully evaluated at present. In a previous study (51), a sub-conjunctival injection with glyceraldehyde resulted in intraocular pressure elevation and loss of retinal ganglion cell axon, which means that this type of SXL induced glaucoma in these eyes.

\section{DISCUSSIONS AND PREDICTIONS}

As scleral crosslinking remains untested on human eyes, we believe that through continuous research and development, SXL would eventually become a novel method for shaping eyeballs and retarding myopic progression. In contrast with the current methods of optometry correction (spectacle works, contact lenses, refractive surgeries, etc.), eyeball-shaping method using SXL would be a fundamental intervention which aim at the pathogenesis of progressive visual loss of myopia. SXL will be also used for preventing and treating the myopia-related complications, such as staphyloma, tractive retinal detachment and macular cleave. Compared with the current posterior scleral reinforcement (PSR) (52), the most advantage of SXL is that there is no allotransplant into the myopic eye, which means less expenditure, lower risk, and easier to handle in operating.

For photochemically-induced crosslinking, several necessary developments on light delivery system will make this SXL method much safer than the current ones. The novel light delivery will provide homogeneous light distribution on scleral spherical surface. This light-delivery system can be easily and flexibly introduced and operated deep in the eye socket to deliver light to the relatively large target region with sufficient irradiance, short procedural time and reduced invasiveness. For accuracy, this system can provide a series of irradiation area size, from several square millimeters to hundred square millimeters, without any extra light leakage to the adjacent tissues. For safety, this system can create its SXL effects on the outer and medium layers of sclera, and can be no harmful for the deeper ocular tissues.

For chemically-induced crosslinking, outstanding crosslinking agents will be selected according to their efficacy under physiologic $\mathrm{pH}$ and temperature, permeability, and cell toxicity (53). Mature treatment strategies will be set up and used including the drug compositions, dose, injection sites, and repetition frequency based on the illness condition. Novel drug delivery systems, such as microneedles (54), sub-tenon (episcleral) implants (55), and transscleral iontophoresis (56), 
will be combined for increasing the bioavailability of crosslinking agents, improving their penetrability, reducing their systemic absorption and side effects, reducing administration frequency, and improving the comfort and compliance of patients.

Until now, the current SXL protocol has a lot of urgent questions to be answered. For example, dose-effect relationship and dose-toxicity relationship should be generalized. For photochemically-induced crosslinking, the light delivery systems should be initially optimized for less damage. For chemically-induced crosslinking, both effective and safe chemical crosslinking agents should be established using more comparative studies. Long-term observation of SXL efficacy should also be lasting for several years. Thus, there is still a long road ahead before clinical studies are conducted.

Because of their respective characteristics, both photochemically-induced crosslinking and chemically-induced crosslinking will be performed according to the myopic types and the treatment plan. Photochemically-induced crosslinking, which can be easily controlled in dosage, duration and area size of irradiation, will be applied on the symmetrical locations of sclera for axial myopia or on the partial sclera for staphyloma. For larger and homogeneous crosslinking effect, chemically-induced crosslinking can be repeatedly applied by multi-site injections around the equatorial sclera or even the whole sclera. Moreover, it should be noticed that SXL can only stabilize the situation of myopia but not improve vision. This means that SXL can only be useful on eyes while myopia is progressing but not when myopia has stabilized. Thus, we believe that a complex series of

\section{REFERENCES}

1. Kumar A, Chawla R, Kumawat D, Pillay G. Insight into high myopia and the macula. Indian J Ophthalmol. (2017) 65:85-91. doi: 10.4103/ijo.IJO_863_16

2. Holden BA, Fricke TR, Wilson DA, Jong $M$, Naidoo KS, SankaridurgP, et al. Global prevalence of myopia and high myopia and temporal trends from 2000 through 2050. Ophthalmology. (2016) 123:1036-42. doi: 10.1016/j.ophtha.2016.01.006

3. Ohno-Matsui K, Lai TYY, Lai C,Cheung CMG. Updates of pathologic myopia. Prog Retin Eye Res. (2016) 52:156-87. doi: 10.1016/j.preteyeres.2015.12.001

4. Chan NS, Teo K, Cheung CMG. Epidemiology and diagnosis of myopic choroidal neovascularization in asia. Eye Contact Lens. (2016) 42:4855. doi: 10.1097/ICL.0000000000000201

5. Saw S, Gazzard G, Shih-Yen EC,Chua W. Myopia and associated pathological complications. Ophthalmic Physiol Opt. (2005) 25:381-91. doi: 10.1111/j.1475-1313.2005.00298.x

6. Stone RA, Flitcroft DI. Ocular shape and myopia. Ann Acad Med Singap. (2004) 33:7-15

7. Wenbo L, Congxia B, Hui L. Genetic and environmental-genetic interaction rules for the myopia based on a family exposed to risk from a myopic environment. Gene. (2017) 626:305-8. doi: 10.1016/j.gene.2017.05.051

8. Hammond CJ, Snieder H, Gilbert CE, Spector TD. Genes and environment in refractive error: the twin eye study. Invest Ophthalmol Vis Sci. (2001) 42:1232-6.

9. McBrien NA, Gentle A. Role of the sclera in the development and pathological complications of myopia. Prog Retin Eye Res. (2003) 22:30738. doi: 10.1016/S1350-9462(02)00063-0

10. Wollensak G, Spoerl E. Collagen crosslinking of human and porcine sclera. $J$ Cataract Refract Surg. (2004) 30:689-95. doi: 10.1016/j.jcrs.2003.11.032

11. O'Brart DPS. Corneal collagen crosslinking for corneal ectasias: a review. Eur J Ophthalmol. (2017) 27:253-69. doi: 10.5301/ejo.5000916 treatment, which includes not only both kinds of SXLs but also adding refractive corrections (spectacle works, contact lenses, refractive surgeries, etc.) and myopia-related complication treatments (photodynamic therapy, laser photocoagulation, anti-VEGF intravitreal injections, pars plana vitrectomy, etc.) will be applied in clinics according to the realistic conditions and treatment needs in the future.

\section{DATA AVAILABILITY STATEMENT}

The original contributions presented in the study are included in the article/supplementary material, further inquiries can be directed to the corresponding author/s.

\section{AUTHOR CONTRIBUTIONS}

MW and FZ collected the data as well as drafted and revised the manuscript. CC revised the manuscript. MW conceptualized and designed the hypothesis as well as reviewed the manuscript. All authors approved the final manuscript as submitted and agreed to be accountable for all aspects of the work.

\section{FUNDING}

This study was supported by Grants from National Natural Science Foundation of China (MW: 81700873; FZ: 81570877) and Hebei Province Science and Technology Support Program (MW: 18277754D).
12. Rada JAS, Shelton S, Norton TT. The sclera and myopia. Exp Eye Res. (2006) 82:185-200. doi: 10.1016/j.exer.2005.08.009

13. Baldivia S, Levy A, Hegde S, Aper SJA, Merkx M, Grytz R. A novel organ culture model to quantify collagen remodeling in tree shrew sclera. PLoS ONE. (2016) 11:e0166644. doi: 10.1371/journal.pone.0166644

14. Chen M, Dai J, Chu R, Qian Y. The efficacy and safety of modified snyder-Thompson posterior scleral reinforcement in extensive high myopia of chinese children. Graefes Arch Clin Exp Ophthalmol. (2013) 251:26338. doi: 10.1007/s00417-013-2429-x

15. Elsheikh A, Wang D, Brown M, Rama P, Campanelli M, Pye D. Assessment of corneal biomechanical properties and their variation with age. Curr Eye Res. (2007) 32:11-9. doi: 10.1080/02713680601077145

16. McBrien NA, Norton TT. Prevention of collagen crosslinking increases form-deprivation myopia in tree shrew. Exp Eye Res. (1994) 59:47586. doi: 10.1006/exer.1994.1133

17. Sady C, Khosrof S, Nagaraj R. Advanced maillard reaction and crosslinking of corneal collagen in diabetes. BiochemBiophys Res Commun. (1995) 214:793-7. doi: 10.1006/bbrc.1995. 2356

18. Løgstrup N, Sjølie AK, Kyvik KO, Green A. Long-term influence of insulin dependent diabetes mellitus on refraction and its components: a population based twin study. Br J Ophthalmol. (1997) 81:343-9. doi: 10.1136/bjo.81. 5.343

19. Awetissow ES. [The role of the sclera in the pathogenesis of progressive myopia (author's transl)]. KlinMonblAugenheilkd. (1980) 176:777-81. doi: 10.1055/s-2008-105 7552

20. Kamaev P, Friedman MD, Sherr E, Muller D. Photochemical kinetics of corneal cross-linking with riboflavin. Invest Ophthalmol Vis Sci. (2012) 53:2360-7. doi: 10.1167/iovs.119385 
21. Raiskup F, Spoerl E. Corneal crosslinking with riboflavin and ultraviolet a. I. Principles. Ocul Surf. (2013) 11:65-74. doi: 10.1016/j.jtos.2013.01.002

22. Li Y, Zhang F, Sun M, Lai L, LvX, Liu C, et al. Safety and long-term scleral biomechanical stability of rhesus eyes after scleral cross-linking by blue light. Curr Eye Res. (2021) 20:1-10. doi: 10.1080/02713683.2020.1853781

23. Wollensak G, Redl B. Gel electrophoretic analysis of corneal collagen after photodynamic cross-linking treatment. Cornea. (2008) 27:353-6. doi: 10.1097/ICO.0b013e31815cf66a

24. Wollensak G, Iomdina E. Long-term biomechanical properties after collagen crosslinking of sclera using glyceraldehyde. Acta Ophthalmol. (2008) 86:88793. doi: 10.1111/j.1755-3768.2007.01156.x

25. Avila MY, Navia JL. Effect of genipin collagen crosslinking on porcine corneas. J Cataract Refract Surg. (2010) 36:659-64. doi: 10.1016/j.jcrs.2009.11.003

26. Liu T, Luo X, Gu Y, Yang B, Wang Z. Correlation of discoloration and biomechanical properties in porcine sclera induced by genipin. Int $J$ Ophthalmol. (2014) 7:621-5. doi: 10.3980/j.issn.2222-3959.2014.04.06

27. Liu T, Wang Z. Collagen crosslinking of porcine sclera using genipin. Acta Ophthalmol. (2013) 91:e253-7. doi: 10.1111/aos.12172

28. Spoerl E, Boehm AG, Pillunat LE. The influence of various substances on the biomechanical behavior of lamina cribrosa and peripapillary sclera. Invest Ophthalmol Vis Sci. (2005) 46:1286-90. doi: 10.1167/iovs.04-0978

29. Wong FF, Lari DR, Schultz DS, Stewart JM. Whole globe inflation testing of exogenously crosslinked sclera using genipin and methylglyoxal. Exp Eye Res. (2012) 103:17-21. doi: 10.1016/j.exer.2012.06.010

30. Babar N, Kim M, Cao K, Shimizu Y, Kim S, Takaoka A, et al. Cosmetic preservatives as therapeutic corneal and scleral tissue cross-linking agents. Invest Ophthalmol Vis Sci. (2015) 56:1274-82. doi: 10.1167/iovs.14-16035

31. Zyablitskaya M, Takaoka A, Munteanu EL, Nagasaki T, Trokel SL, Paik DC. Evaluation of therapeutic tissue crosslinking (TXL) for myopia using second harmonic generation signal microscopy in rabbit sclera. Invest Ophthalmol Vis Sci. (2017) 58:21-9.doi: 10.1167/iovs.16-20241

32. Campbell IC, Hannon BG, Read AT, Sherwood JM, Schwaner SA, Ethier CR. Quantification of the efficacy of collagen cross-linking agents to induce stiffening of rat sclera. J $R$ Soc Interface. (2017) 14:20170014. doi: 10.1098/rsif.2017.0014

33. Lin X, Naidu RK, Dai J,Zhou X, Qu X, Zhou H. Scleral cross-Linking using glyceraldehyde for the prevention of axial elongation in the rabbit: blocked axial elongation and altered scleral microstructure. Curr Eye Res. (2019) 44:162-71. doi: 10.1080/02713683.2018.1522647

34. Kymionis GD, Mikropoulos DG, Portaliou DM, Voudouragkaki IC, Kozobolis VP, Konstas AGP. An overview of corneal collagen cross-linking (CXL). Adv Ther. (2013) 30:858-69. doi: 10.1007/s12325-013-0065-9

35. Wollensak G, Spoerl E, Seiler T. Riboflavin/ultraviolet-a-induced collagen crosslinking for the treatment of keratoconus. Am J Ophthalmol. (2003) 135:620-7. doi: 10.1016/S0002-9394(02)02220-1

36. Caporossi A, Mazzotta C, Baiocchi S, Bagaglia S, Caporossi O, Villano A, et al. Long-term results of riboflavin ultraviolet a corneal collagen cross-linking for keratoconus in italy: the siena eye cross study. Am J Ophthalmol. (2010) 149:585-93. doi: 10.1016/j.ajo.2009.10.021

37. Kanellopoulos AJ, Binder PS. Management of corneal ectasia after IASIK with combined, same-day, topography-guided partial transepithelial pRK and collagen cross-linking: the athens protocol. J Refract Surg. (2011) 27:32331. doi: 10.3928/1081597X-20101105-01

38. Xiao B, Chu Y, Wang H, Han Q. Minimally invasive repetitive uVA irradiation along with riboflavin treatment increased the strength of sclera collagen crossLinking. J Ophthalmol. (2017) 2017:1324012. doi: 10.1155/2017/1324012

39. Wang M, Corpuz CCC. Effects of scleral cross-linking using genipin on the process of form-deprivation myopia in the guinea pig: a randomized controlled experimental study. BMC Ophthalmol. (2015) 15:89. doi: 10.1186/s12886-015-0086-Z

40. Chu Y, Cheng Z, Liu J, Wang Y, Guo H, Han Q. The effects of scleral collagen cross-Linking using glyceraldehyde on the progression of form-Deprived myopia in guinea pigs. J Ophthalmol. (2016) 2016:3526153. doi: 10.1155/2016/3526153

41. Wollensak G, Iomdina E. Long-term biomechanical properties of rabbit sclera after collagen crosslinking using riboflavin and ultraviolet a (UVA). Acta Ophthalmol. (2009) 87:193-8. doi: 10.1111/j.1755-3768.2008.01229.x
42. Sun M, Zhang F, Li Y, Ouyang B, Wang M, Jiao X, et al. Evaluation of the safety and long-term scleral biomechanical stability of uVA cross-linking on scleral collagen in rhesus monkeys. $J$ Refract Surg. (2020) 36:696-702. doi: 10.3928/1081597X-202008 07-01

43. Gawargious BA,Le A, Lesgart M, UgradarS, Demer JL. Differential regional stiffening of sclera by collagen cross-linking. Curr Eye Res. (2020) 45:718-25. doi: 10.1080/02713683.2019.169 4157

44. Choi S, Lee S, Lee H, Cheong Y, Jung G, JinK, et al. Structural response of human corneal and scleral tissues to collagen cross-linking treatment with riboflavin and ultraviolet a light. Lasers Med Sci. (2013) 28:1289-96. doi: 10.1007/s10103-012-1 237-6

45. Karl A, Makarov FN, Koch C, KörberN, SchuldtC, Krüger M, et al. The ultrastructure of rabbit sclera after scleral crosslinking with riboflavin and blue light of different intensities. Graefes Arch Clin Exp Ophthalmol. (2016) 254:1567-1577. doi: 10.1007/s00417-016-3 393-z

46. Wollensak G, Iomdina E, Dittert D, Salamatina O, Stoltenburg G. Crosslinking of scleral collagen in the rabbit using riboflavin and uVA. Acta Ophthalmol Scand. (2005) 83:477-82. doi: 10.1111/j.1600-0420.2005.00447.x

47. Zhang X, Tao X, Zhang J, Li ZW, Xu YY, Wang YM, et al. A review of collagen cross-linking in cornea and sclera. J Ophthalmol. (2015) 2015:289467. doi: 10.1155/2015/289467

48. Kwok SJJ, Kim M, Lin HH, Seiler TG, Beck E, ShaoP, et al. Flexible optical waveguides for uniform periscleral cross-Linking. Invest Ophthalmol Vis Sci. (2017) 58:2596-602. doi: 10.1167/iovs.17-21559

49. Kwok SJJ, Forward S, Wertheimer CM, Liapis AC, Lin HH, Kim M, et al. Selective equatorial sclera crosslinking in the orbit using a metalCoated polymer waveguide. Invest Ophthalmol Vis Sci. (2019) 60:256370. doi: 10.1167/iovs.19-26709

50. Kumar CM, McNeela BJ. Ultrasonic localization of anaesthetic fluid using sub-Tenon's cannulae of three different lengths. Eye (Lond). (2003) 17:10037. doi: 10.1038/sj.eye.6700501

51. Kimball EC, Nguyen C, Steinhart MR, Nguyen TD, Pease ME, Oglesby EN, et al. Experimental scleral cross-linking increases glaucoma damage in a mouse model. Exp Eye Res. (2014) 128:129-40. doi: 10.1016/j.exer.2014.08.016

52. Huang W, Duan A, Qi Y. Posterior scleral reinforcement to prevent progression of high myopia. Asia Pac J Ophthalmol (Phila). (2019) 8:36670. doi: 10.1097/APO.0000000000000257

53. Takaoka A, Cao K, Oste EM, Nagasaki T, Paik DC. Topical therapeutic corneal and scleral tissue cross-linking solutions: in vitro formaldehyde release studies using cosmetic preservatives. Biosci Rep. (2019) 39:BSR20182392. doi: 10.1042/BSR20182392

54. Gupta P, Yadav KS. Applications of microneedles in delivering drugs for various ocular diseases. Life Sci. (2019) 237:116907. doi: 10.1016/j.lfs.2019.116907

55. Zhou C, Robert M, Kapoulea V, Lei F, Stagner AM, Jakobiec FA, et al. Sustained subconjunctival delivery of infliximab protects the cornea and retina following alkali burn to the eye. Invest Ophthalmol Vis Sci. (2017) 58:96-105. doi: 10.1167/iovs.16-20339

56. Perez VL, Wirostko B, Korenfeld M, From S, Raizman M. Ophthalmic drug delivery using iontophoresis: recent clinical applications. J Ocul Pharmacol Ther. (2020) 36:75-87. doi: 10.1089/jop.2019.0034

Conflict of Interest: The authors declare that the research was conducted in the absence of any commercial or financial relationships that could be construed as a potential conflict of interest.

Copyright (c) 2021 Wang, Corpuz and Zhang. This is an open-access article distributed under the terms of the Creative Commons Attribution License (CC BY). The use, distribution or reproduction in other forums is permitted, provided the original author(s) and the copyright owner(s) are credited and that the original publication in this journal is cited, in accordance with accepted academic practice. No use, distribution or reproduction is permitted which does not comply with these terms. 